\title{
GMR
}

\section{Polymorphism analysis of multi-parent advanced generation inter-cross (MAGIC) populations of upland cotton developed in China}

\author{
D.G. Li ${ }^{1 *}$, Z.X. Li ${ }^{1 *}$, J.S. Hu ${ }^{1}$, Z.X. $\operatorname{Lin}^{2}$ and X.F. Li ${ }^{1}$ \\ ${ }^{1}$ Institute of Crop Genetic and Breeding, Yangtze University, Jingzhou, \\ Hubei Province, China \\ ${ }^{2}$ National Key Laboratory of Crop Genetic Improvement, \\ College of Plant Science \& Technology of Huazhong Agricultural University, \\ Wuhan, Hubei Province, China \\ *These authors contributed equally to the study. \\ Corresponding authors: X.F. Li / Z.X. Lin \\ E-mail: lixiaofang@163.net / linzhongxu@mail.hzau.edu.cn.
}

Genet. Mol. Res. 15 (4): gmr15048759

Received May 4, 2016

Accepted November 1, 2016

Published December 19, 2016

DOI http://dx.doi.org/10.4238/gmr15048759

Copyright $(C 2016$ The Authors. This is an open-access article distributed under the terms of the Creative Commons Attribution ShareAlike (CC BY-SA) 4.0 License.

\begin{abstract}
Upland cotton (Gossypium hirsutum L.) is an important cash crop that provides renewable natural fiber worldwide. Currently limited genetic base leads to a decrease in upland cotton genetic diversity. Multi-parent advance generation inter-cross (MAGIC) populations can be used to evaluate complex agronomic traits in crops. In this study, we developed an upland cotton MAGIC population. A total of 258 MAGIC population lines and their twelve founder lines were analyzed, using 432 pairs of simple sequence repeat (SSR) markers. Gene diversity indices and the polymorphism information content
\end{abstract}

Genetics and Molecular Research 15 (4): gmr15048759 
were calculated using polymorphism analyses. Our genotype analysis showed that 258 inbred lines could be divided into 158 genotypes. Among these, we identified 17 pairs of specific SSR primers on the A chromosome subgroups and 24 pairs of specific SSR primers on the B chromosome subgroups of upland cotton. These were related to 77 and 128 genotypes, respectively. Our results suggest that the upland cotton MAGIC population contained abundant genetic diversity and may provide enormous resources for future genetic breeding.

Key words: Upland cotton; MAGIC; SSR; Genetic diversity

\section{INTRODUCTION}

Domesticated upland cotton (Gossypium hirsutum L.) is one of the world's leading cash crops. It constitutes the largest source of renewable natural textile fiber in China. Genetic improvement of fiber yield and quality are the primary objectives of cotton breeding programs (Pan, 1998). However, the genetic base of upland cotton cultivars is narrow (Iqbal et al., 1997; Tu et al., 2014), which leads to a decrease in upland cotton genetic diversity. In order to increase the intravariety genetic diversity based on existing germplasm resources, multi-parent advance generation inter-cross (MAGIC), a new breeding method using multiple parents' convergent cross, has been widely used to improve the genetic diversity of crops (Li, 2014; Cavanagh et al., 2008).

Many major agronomic traits are controlled by multiple genetic loci, which makes it difficult to study multiple complicated agronomic traits in bi-parental or natural populations (Wang et al., 2016). MAGIC populations, including multiple parents and alleles, have been used for linkage mapping and association analyses without the limitations associated with structured populations (Sallam and Martsch, 2015). A MAGIC mapping population could enhance the mapping accuracy and resolution of any identified quantitative trait loci (QTLs) (Valdar et al., 2006). There is currently ample evidence supporting the value of MAGIC populations for gene mapping and crop breeding ( $\mathrm{Li}$ et al., 2013; Wei and $\mathrm{Xu}$, 2016). For example, MAGIC populations have been developed and used for construction of efficient genetic maps in wheat, Arabidopsis thaliana, and rice (Kover et al., 2009; Huang et al., 2012; Li et al., 2013).

Given the potential benefits of MAGIC populations, the first upland cotton MAGIC populations using pest-resistant upland cotton ecotypes could be developed to analyzed polymorphic simple sequence repeat (SSR) markers, which might provide germplasm resources for future upland cotton breeding.

\section{MATERIAL AND METHODS}

\section{Parent materials and growing environment}

The twelve founders included breeder-relevant germplasms with high yield and pest-, Fusarium wilt-, and Verticillium wilt-resistant varieties (Table 1). Detailed information about the parent materials is presented in Table 1. To construct the MAGIC population of upland cotton, the different parent material were planted at Yangtze University $\left(112^{\circ} \mathrm{E}, 30^{\circ} \mathrm{N}\right)$. Common urea $(\mathrm{N}, 20 \mathrm{~kg})$, calcium superphosphate $\left[\mathrm{Ca}\left(\mathrm{H}_{2} \mathrm{PO}_{4}\right)_{2}, 50 \mathrm{~kg}\right]$, and potassium sulfate $\left(\mathrm{K}_{2} \mathrm{SO}_{4}, 15\right.$ $\mathrm{kg}$ ) were applied as base fertilizers.

Genetics and Molecular Research 15 (4): gmr15048759 
Table 1. List of parent materials of upland cotton (Gossypium hirsutum).

\begin{tabular}{l|l|l|l}
\hline No. & Name & Material sources & Agronomic traits \\
\hline P1 & GY4 & Agricultural College of Yangtze University & High yield \\
\hline P2 & GY5 & Agricultural College of Yangtze University & High yield \\
\hline P3 & GY6 & Agricultural College of Yangtze University & High yield \\
\hline P4 & GY7 & Hebei Academy of Agriculture and Forestry & High yield \\
\hline P5 & GY2 & Taicang City cotton seed farm in Jiangsu Province & High yield \\
\hline P6 & CQ9 & Chinese Cotton Research Institute of Academy of Agricultural Sciences & Pest-resistant \\
\hline P7 & CQ13 & Chinese Cotton Research Institute of Academy of Agricultural Sciences & Pest-resistant \\
\hline P8 & CQ2 & Hubei province seed management station & Pest-resistant \\
\hline P9 & KC9 & Hubei province seed management station & Pest-resistant \\
\hline P10 & GY8 & Chinese Cotton Research Institute of Academy of Agricultural Sciences & Fusarium wilt-resistant and Verticillium wilt-resistant \\
\hline P11 & KB8 & Chinese Cotton Research Institute of Academy of Agricultural Sciences & Fusarium wilt-resistant and Verticillium wilt-resistant \\
\hline P12 & KB10 & Chinese Cotton Research Institute of Academy of Agricultural Sciences & Fusarium wilt-resistant and Verticillium wilt-resistant \\
\hline
\end{tabular}

\section{Construction of the MAGIC population}

According to a three-stage process including mixing, maintenance, and inbreeding (Churchill et al., 2004; Li, 2014), a MAGIC population of upland cotton was developed. As shown in Figure 1, a total of 12 inbred lines were intercrossed to develop the foundation population. Six F1 populations were produced using the following two-way crosses; A (P5 x P11), B (P7 x P2), C (P10 x P12), D (P8 x P9), E (P3 x P6), and F (P1 x P4), respectively. Subsequently, three four-way crosses; $\mathrm{G}$ (female group), H (male group 1), and I (male group 2 ), were identified (Figure 1). Plants from the four-cross group $G$ were then used as females that were crossed with mixed pollen of each pair of the four-cross groups $\mathrm{H}$ and I. Following the method described above, 167 cotton hybrids were obtained. Each hybrid formed a family with 10 plants. Based on an F7 selfing population, 1500 recombinant inbred lines (RILs) were constructed using the single seed descent method (Li, 2014). Finally, 258 lines were selected as experimental materials used in this study.

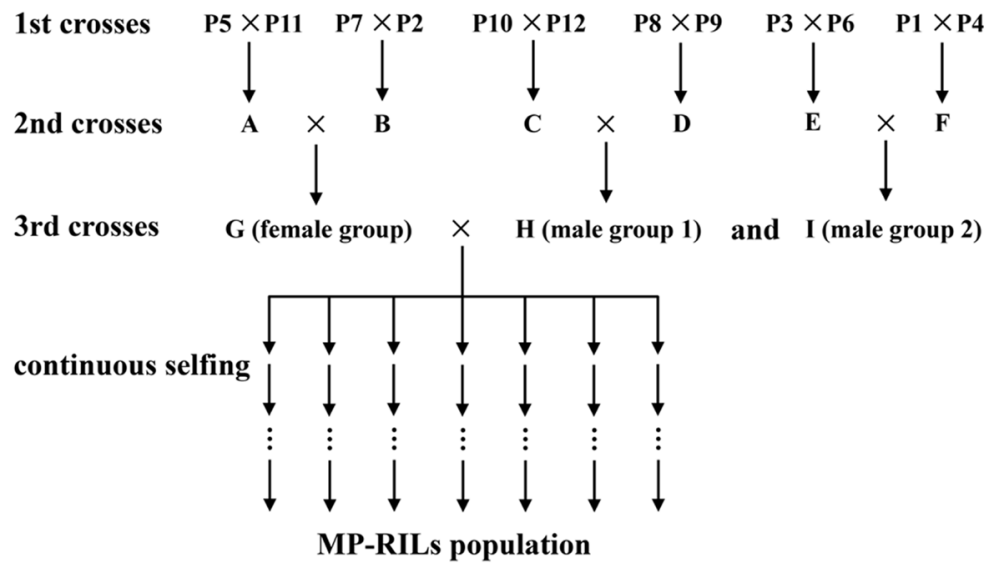

Figure 1. Graphical representation of the construction of the MAGIC population. Twelve founder accessions (P1 to P12) were mated pair-wise to produce six single crosses A, B, C, D, E, and F in the first season. Subsequently, three double-crosses $(\mathrm{G}, \mathrm{H}$, and I) were made in the second season. In the third season, multiple plants of $\mathrm{G}$ were used as females to cross with mixed pollens collected from each pair of $\mathrm{H}$ (male group 1) and I (male group 2) plants. The progeny of the complex crosses was then advanced by 7 generations of selfing.

Genetics and Molecular Research 15 (4): gmr15048759 


\section{SSR marker based genotyping of upland cotton}

Total DNA was extracted from fresh leaves of different cotton varieties using the CTAB method (Song et al., 1998). Following the method of Chen and Du (2006), 436 primer pairs were distributed evenly among 26 linkage group in upland cotton. Among these primers, 42 polymorphic markers were selected for genotyping the 258 mid-parental-RILs and 12 founder lines (P1-P12) (Tables 2 and 3). The information for primer sequences were obtained from CottonGen (https://www.cottongen.org). Polymerase chain reaction (PCR) amplification was performed in a $10-\mu \mathrm{L}$ total volume containing $2.5 \mu \mathrm{L}$ template, $1 \mu \mathrm{L} 10 \mathrm{X}$ PCR buffer (with $\left.\mathrm{Mg}^{2+}\right), 0.15 \mu \mathrm{L}$ Taq DNA polymerase, $10 \mathrm{mM}$ dNTPs, $7.48 \mu \mathrm{L} \mathrm{ddH}_{2} \mathrm{O}$, and $0.16 \mu \mathrm{L}$ each primer.

Table 2. Detailed information of 42 SSR markers used for genotyping the MAGIC population and 12 founders.

\begin{tabular}{|c|c|c|c|c|c|}
\hline No. & Name & Chromosome locus & Allele frequency and No. & Gene diversity index & PIC value* \\
\hline 1 & TMB1738 & Chr.2 & $0.922(3)$ & 0.146 & 0.140 \\
\hline 2 & NAU3541 & Chr.3 & $0.800(4)$ & 0.323 & 0.275 \\
\hline 3 & JESPR231 & Chr.3 & $0.693(5)$ & 0.445 & 0.372 \\
\hline 4 & NAU4034 & Chr.5 & $0.978(3)$ & 0.044 & 0.043 \\
\hline 5 & NAU3036 & Chr.5 & $0.970(4)$ & 0.058 & 0.057 \\
\hline 6 & NAU5387 & Chr.5 & $0.982(4)$ & 0.037 & 0.036 \\
\hline 7 & BNL1664 & Chr. 8 & $0.704(3)$ & 0.427 & 0.351 \\
\hline 8 & HAU0356 & Chr.8 & $0.996(2)$ & 0.007 & 0.007 \\
\hline 9 & HAU1966 & Chr.9 & $0.600(2)$ & 0.480 & 0.365 \\
\hline 10 & NAU859 & Chr.9 & $0.619(4)$ & 0.505 & 0.420 \\
\hline 11 & BNL3031 & Chr.9 & $0.900(4)$ & 0.183 & 0.171 \\
\hline 12 & JESPR29 & Chr.9 & $0.459(4)$ & 0.594 & 0.510 \\
\hline 13 & NAU3390 & Chr.11 & $0.985(3)$ & 0.029 & 0.029 \\
\hline 14 & Gh329 & Chr.11 & $0.967(4)$ & 0.065 & 0.064 \\
\hline 15 & NAU1148 & Chr.11 & $0.696(3)$ & 0.432 & 0.350 \\
\hline 16 & BNL598 & Chr.12 & $0.978(4)$ & 0.044 & 0.043 \\
\hline 17 & Gh697 & Chr.13 & $0.670(3)$ & 0.451 & 0.362 \\
\hline 18 & BNL1652 & Chr.13 & $0.511(4)$ & 0.546 & 0.445 \\
\hline 19 & HAU1951 & Chr.14 & $0.996(2)$ & 0.007 & 0.007 \\
\hline 20 & Gh471 & Chr.14 & $0.622(4)$ & 0.503 & 0.417 \\
\hline 21 & JESPR156 & Chr.14 & $0.989(2)$ & 0.022 & 0.022 \\
\hline 22 & TMB0071 & Chr.14 & $0.956(3)$ & 0.086 & 0.084 \\
\hline 23 & HAU2489 & Chr.15 & $0.948(2)$ & 0.098 & 0.094 \\
\hline 24 & TMB2295 & Chr.18 & $0.600(4)$ & 0.527 & 0.445 \\
\hline 25 & NAU3943 & Chr.18 & $0.993(3)$ & 0.015 & 0.015 \\
\hline 26 & MUSB1135 & Chr.18 & 0.559 (3) & 0.515 & 0.406 \\
\hline 27 & NAU3497 & Chr.19 & $0.682(4)$ & 0.447 & 0.366 \\
\hline 28 & NAU3405 & Chr.19 & $0.989(2)$ & 0.022 & 0.022 \\
\hline 29 & NAU2894 & Chr.19 & $0.352(7)$ & 0.744 & 0.701 \\
\hline 30 & NAU5389 & Chr.21 & $0.630(4)$ & 0.506 & 0.428 \\
\hline 31 & NAU2873 & Chr.23 & $0.904(3)$ & 0.178 & 0.169 \\
\hline 32 & HAU3241 & Chr.23 & $0.522(3)$ & 0.513 & 0.396 \\
\hline 33 & TMB0913 & Chr.23 & $0.619(3)$ & 0.519 & 0.443 \\
\hline 34 & BNL3140 & Chr.23 & $0.922(4)$ & 0.147 & 0.143 \\
\hline 35 & CIR388 & Chr.24 & $0.585(3)$ & 0.492 & 0.378 \\
\hline 36 & NAU2773 & Chr.25 & $0.596(5)$ & 0.548 & 0.478 \\
\hline 37 & NAU3502 & Chr.25 & $0.982(4)$ & 0.037 & 0.036 \\
\hline 38 & CIR407 & Chr.25 & $0.541(4)$ & 0.516 & 0.404 \\
\hline 39 & TMB2377 & Chr.25 & $0.482(3)$ & 0.548 & 0.444 \\
\hline 40 & MGHES44 & Chr.26 & $0.600(3)$ & 0.516 & 0.426 \\
\hline 41 & HAU1081 & Chr.26 & $0.515(3)$ & 0.550 & 0.451 \\
\hline 42 & DPL0183b & Chr.26 & $0.944(4)$ & 0.107 & 0.104 \\
\hline Mean & & & $0.761(3)$ & 0.309 & 0.260 \\
\hline
\end{tabular}

*PIC = polymorphism information content.

Genetics and Molecular Research 15 (4): gmr15048759 
The PCR cycle profile was as follows: $94^{\circ} \mathrm{C}$ for $3 \mathrm{~min} ; 35$ cycles of $94^{\circ} \mathrm{C}$ for $40 \mathrm{~s}$, 50 to $62^{\circ} \mathrm{C}$ for $45 \mathrm{~s}, 72^{\circ} \mathrm{C}$ for $40 \mathrm{~s}$, and $72^{\circ} \mathrm{C}$ for $5 \mathrm{~min}$. PCR products were separated on a $6 \%$ denaturing polyacrylamide gel at $8 \mathrm{~V} / \mathrm{cm}$ for 2 to $3 \mathrm{~h}$ and then silver stained as previously described (Xu et al., 2002). According to their sizes on the gel, the allelic bands amplified by each SSR primer pair were labeled A, B, C, etc.

Table 3. Primer sequences used for PCR analysis.

\begin{tabular}{|c|c|c|c|}
\hline No. & Accession No. & Primer sequence $\left(5^{\prime}-3^{\prime}\right)$ and $\operatorname{Tm}^{*}\left({ }^{\circ} \mathrm{C}\right)$ & Reverse primer sequence $\left(5^{\prime}-3^{\prime}\right)$ and $\operatorname{Tm} *\left({ }^{\circ} \mathrm{C}\right)$ \\
\hline 1 & TMB1738 & TTGTTAGCAATATGCAATATGAAC (55.3) & GGGTTTAGTTGAATGGGACC (56) \\
\hline 2 & NAU3541 & ATCCCACATTGCCTTCTTAT (53.8) & CTTTCСССТCTCAAATACCC (55.5) \\
\hline 3 & JESPR231 & GCTGGTGGGATTCTCTG (51.6) & CTATGAACTGCTGGCTATGG (53.4) \\
\hline 4 & NAU4034 & CGACGGAAAGGGTTATCTTA (54.9) & ACGCCCTTCATTCAAACAC (54.1) \\
\hline 5 & NAU3036 & ATCTTGGGAATCTCAAATGG (54) & TGCTCCGATGAGTATTCAAA (54) \\
\hline 6 & NAU5387 & TTGGTCTTGCTCTCTTCCTC (54.2) & TCGTGTGAATGAAGCCTAAA (54.2) \\
\hline 7 & BNL1664 & ATTGCAAACGAGTGGAGAGC (57.7) & TGGATTCCAAGGCATTTTGT (57.9) \\
\hline 8 & HAU0356 & GATGCCCCTTCTCTAGCATA (54.6) & ACTCACGCAAAGCATACAAA (54.3) \\
\hline 9 & HAU1966 & CCCCCTTCAGATATCTTCATT (55.2) & CAGCCCAAAACAGCTAAAAT (55.2) \\
\hline 10 & NAU859 & CAGGCTTCATCTTTTTGGAC (54.8) & CATTGGATCCTAGTGGGAAG (54.4) \\
\hline 11 & BNL3031 & AGGCTGACCCTTTAAGGAGC (57.9) & AACCAACTTTTCCAACACCG (57.5) \\
\hline 12 & JESPR29 & CACCGTTTCCAAGTAAGATT (52.1) & GGTTAATCTTAGTTGAGGTC (45.6) \\
\hline 13 & NAU3390 & GAAAACAAGGCACTTGAACC (54.8) & AAACCGGTAACGACAACAAT (54.8) \\
\hline 14 & Gh329 & CAGCAGGCAGAAATCTTGTGATCG (66) & СТTAАATTТСТСТСССТСАAАACCATC (60.8) \\
\hline 15 & NAU1148 & AGGGTTTGCAGTGGATTAAA (55) & CCAAAAGGATAGTAGCATGGA (54.9) \\
\hline 16 & BNL598 & TATCTCCTTCACGATTCCATCAT (58.1) & AAAAGAAAACAGGGTCAAAAGAA (57.5) \\
\hline 17 & Gh697 & TCCCTGAGCTCATATCTAACTTCC (59) & GACTTACTAAGCTATTCAAGCTTCC (56.2) \\
\hline 18 & BNL1652 & CACGGCTGAAAGGTGAAATT (57.8) & GCATGTACGCACTTGCACAG (58.2) \\
\hline 19 & HAU1951 & CAAACGCTGTCACTACCAAG (54) & TGACAAAATGTGCTTCCATC (53.8) \\
\hline 20 & Gh471 & CAGGCATCAACTAGCATTGAAAACG (65.2) & ATCTTCTGATCTCTATTAGCTACAACG (52.3) \\
\hline 21 & JESPR156 & GCCTTCAATCAATTCATACG (53.3) & GAAGGAGAAAGCAACGAATTAG (56) \\
\hline 22 & TMB0071 & GGCGGTCCCATGGTAGTAAT (58.9) & CGAACTATGACTCAATCCACC (54.5) \\
\hline 23 & HAU2489 & GGCACGAGGAGAAAATGAAAGA (61.1) & GATCGGATTCTGGGTCCCGC (66) \\
\hline 24 & TMB2295 & TGAGTTCATGTTCCCCACTG (56) & CTAAACATACTCTGTCAAACAC (47) \\
\hline 25 & NAU3943 & TTGGAGCAAACACAGACACT (53.3) & CCCTACGTCTCGAAAATACG (55.3) \\
\hline 26 & MUSB1135 & CCGCCGTCATTTCCATCACC (65.5) & TGGTACGGATCATGGGAATCCT (62.9) \\
\hline 27 & NAU3497 & TTGCTTAGCTGGTGAGACTG (53.7) & CССТTCACCACСТCTTTCTA (54.2) \\
\hline 28 & NAU3405 & AATAGCAAAGCCTTCAGTGC (55.1) & GAAGTGCAAAAACCGTACCT (54.7) \\
\hline 29 & NAU2894 & GGCACGTTGCAAGTGTTAT (53.2) & AACCTTCCAGAGAAAGCAGA (54) \\
\hline 30 & NAU5389 & CCTCCATCGTCAACTCTTCT (53.8) & GCCTTGATCTTTGTCGTTCT (54.3) \\
\hline 31 & NAU2873 & TGTCCCCATGATTCTTCTTT $(54.3)$ & TATTTTCAGCACCAAGAGCA (54.4) \\
\hline 32 & HAU3241 & AGGAATTCAAGGTTGAAGGAGGGT (63.7) & GGCTGCTGCTCAGTCTGCAT (61.1) \\
\hline 33 & TMB0913 & TAATTGCATGGCATCGAAAA (57.7) & GGGTGTGATCCAGACAGTCA (55.4) \\
\hline 34 & BNL3140 & CACCATTGTGGCAACTGAGT (56.1) & GGAAAAGGGAAAGCCATTGT (58.4) \\
\hline 35 & CIR388 & TTAAGCATCCAACAAGG (53.6) & TCCAACTTTTGGTCTATGT (48) \\
\hline 36 & NAU2773 & CCCTTTCAATCACAAAAACC $(54.8)$ & CAAGGGCAGTCAATTTATCA (53.6) \\
\hline 37 & NAU3502 & AAGATCCACAAGAAACTGAAAC (53.1) & GGGGTTTTTCTTCTTTTCGT (55.6) \\
\hline 38 & CIR407 & GCACAGAACATCCATACA (42.4) & TCTCTCTCTCTTTCACACAC (45.8) \\
\hline 39 & TMB2377 & TAGTCCCTTACTTTCAATATTTTATA (52.3) & TGTTTTGGTGTAGTGATATAAACG (55) \\
\hline 40 & MGHES44 & ACCACTTGGGATTGGTTCAA (57.4) & GAGGCCACCACATATCGTTT (57.3) \\
\hline 41 & HAU1081 & TGTCTCCCGTACTCAGTGAA (53.2) & GGCTATGGGGTTACAATCAG (54.7) \\
\hline 42 & DPL0183b & TTATTTCTTCCCTTGCGTTCTG (59.3) & AGAGGTGGCAATGGAGTTCTT (47.6) \\
\hline
\end{tabular}

$* \mathrm{Tm}=$ melting temperature.

\section{Data analysis}

The PIC value is an important index to evaluate genetic diversity in MAGIC populations (Agarwal et al., 2015). The polymorphism information content (PIC) was calculated using the following equation: 


$$
\mathrm{PIC}_{j}=1-\sum P_{i j}^{2}
$$

(Equation 1)

in which $P_{i j}$ is the frequency of allele $j$ for marker $i$ in the population. Gene diversity indices were then calculated based on the PIC values (Smith et al., 1997). The genetic similarity coefficient was obtained using the NTSYS-pc v. 2.1 software, and phylogenic trees were constructed using UPGMA and PowerMarker v. 3.25 software. Major allele frequencies and gene diversity indices were calculated using PowerMarker v. 3.25. The Jaccard coefficient was calculated using the following formula:

$$
D_{i j}=1-\left(B_{i j} / M_{i j}\right)
$$

in which $D_{i j}$ is the genetic distance between genotypes $i$ and $j, B_{i j}$ is the number of amplified bands in the two genotypes $i$ and $j$, and $M_{i j}$ is the total number of amplified bands that were recorded in genotypes $i$ and $j$.

\section{RESULTS}

\section{Polymorphism analysis of the MAGIC population and its founders}

To determine changes in genetic diversity, 42 primer pairs selected from 432 SSR markers were used. The results indicated amplification of a total of 145 polymorphic sites. The number of polymorphic sites per marker ranged from 2-7, with an average of 3.452 (Table 2). Interestingly, the number of polymorphic sites obtained from 20 primers higher than the average value. The results indicate that the best primers were screened as the primers to be used to analyze the genetic polymorphism in MAGIC population and its founders. The PIC ranged from 0.007 to 0.700 . The highest PIC values were observed among 23 markers to compare with the average of SSR marker (about 0.260). This result indicated that the upland cotton core SSR primers were highly polymorphic. Compared with the founders, the amount of marker genotypes decreased in the MAGIC population. Increasing the number of markers might have contributed to the allelic variation in the MAGIC population ( $\mathrm{Li}$ et al., 2013). As shown in Table 2, the percentage of new genotypes was $50 \%$. These results suggest that these genotypes will provide abundant genetic variation for crop breeding as well as genes/QTLs for mapping upland cotton.

The genotype frequencies were dramatically different between the MAGIC population and the founder lines; the three genotypes, $\mathrm{AA}, \mathrm{AB}$, and $\mathrm{BB}$, were detected by marker TMB1738 in both the MAGIC population and the founders (Table 4). In the founder lines, the highest genotype frequency observed was $50.0 \%$, compared to $95.7 \%$ in the MAGIC population. The opposite was observed for the least frequent genotype, which was $16.7 \%$ in the founder lines and only $3.5 \%$ in the MAGIC population (Table 4). For marker NAU2894, four and six genotypes were identified in the founder lines and MAGIC population, respectively. Three genotypes were detected using marker NAU3390 in the founders, whereas only a single genotype was found in the MAGIC population.

Genetics and Molecular Research 15 (4): gmr15048759 
Table 4. Changes in genotype frequency between the MAGIC population and the founder lines.

\begin{tabular}{|c|c|c|c|c|c|c|}
\hline \multirow[t]{2}{*}{ Genotype change } & \multirow[t]{2}{*}{ Marker name } & \multirow[t]{2}{*}{ Genotype* } & \multicolumn{2}{|c|}{ Founder } & \multicolumn{2}{|c|}{ MP-RILs } \\
\hline & & & No. & Percent & No. & Percent \\
\hline \multirow{3}{*}{ No change } & \multirow[t]{3}{*}{$\underline{\text { TMB1738 }}$} & 01 & 2 & 16.7 & 9 & 3.5 \\
\hline & & 10 & 6 & 50.0 & 247 & 95.7 \\
\hline & & 11 & 4 & 33.3 & 2 & 0.8 \\
\hline \multicolumn{3}{|l|}{ Total } & 12 & 100 & 258 & 100 \\
\hline \multirow{6}{*}{ Increase } & \multirow[t]{6}{*}{ NAU2894 } & 010 & 2 & 16.7 & 73 & 28.3 \\
\hline & & 011 & 8 & 66.7 & 87 & 33.7 \\
\hline & & 100 & 1 & 8.3 & 51 & 19.7 \\
\hline & & 111 & 1 & 8.3 & 10 & 3.9 \\
\hline & & 101 & 0 & 0 & 35 & 13.6 \\
\hline & & 110 & 0 & 0 & 2 & 0.8 \\
\hline \multicolumn{3}{|c|}{ Total } & 12 & 100 & 258 & 100 \\
\hline \multirow{3}{*}{ Decrease } & \multirow[t]{3}{*}{ NAU3390 } & 101 & 8 & 66.6 & 258 & 100 \\
\hline & & 110 & 2 & 16.7 & 0 & 0 \\
\hline & & 111 & 2 & 16.7 & 0 & 0 \\
\hline \multicolumn{3}{|c|}{ Total } & 12 & 100 & 258 & 100 \\
\hline \multirow{4}{*}{ Substitution } & \multirow[t]{4}{*}{ BNL598 } & 1010 & 3 & 12.5 & 102 & 39.5 \\
\hline & & 1111 & 8 & 66.7 & 137 & 53.1 \\
\hline & & 1101 & 1 & 8.3 & 0 & 0 \\
\hline & & 1110 & 0 & 0 & 19 & 7.4 \\
\hline Total & & & 12 & 100 & 258 & 100 \\
\hline
\end{tabular}

*The underlined genotypes appeared in both the MAGIC population and the founder lines, whereas the genotypes in bold were different.

To further investigate the genetic similarity in the MAGIC progeny population, the genetic similarity coefficient of the MAGIC population and founder lines was calculated using the Jaccard coefficient. Based on the phylogenic trees, the twelve founders clustered into five clades (Figure 2). The genetic similarity coefficient ranged from 0.524 to 1.000 . When the genetic similarity coefficient was 0.721 , the founders clustered into five clades, suggesting that the genetic similarity coefficient of the parental materials of the multi-genotype population was high but that the genetic diversity was narrow (Figure 2). Furthermore, the genetic similarity coefficient of the MAGIC population ranged from 0.515 to 1.000 , with an average of 0.758 . When the genetic similarity coefficient was 0.704 , MAGIC population were clustered into four clades (Figure 3). These results indicate that the genetic diversity of the materials was narrow, and it is possible that many of the local materials were selected among the parental materials.

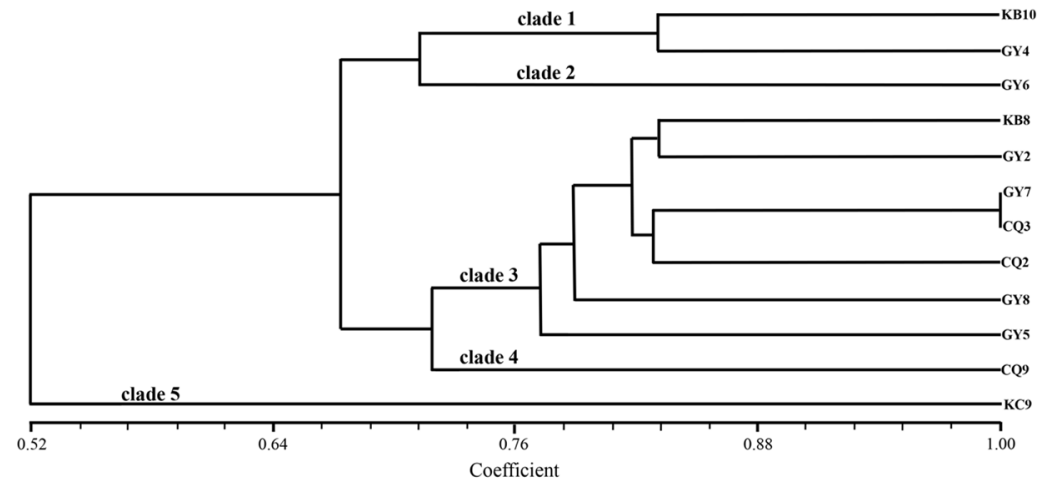

Figure 2. Genotype cluster analysis for the founders.

Genetics and Molecular Research 15 (4): gmr15048759 


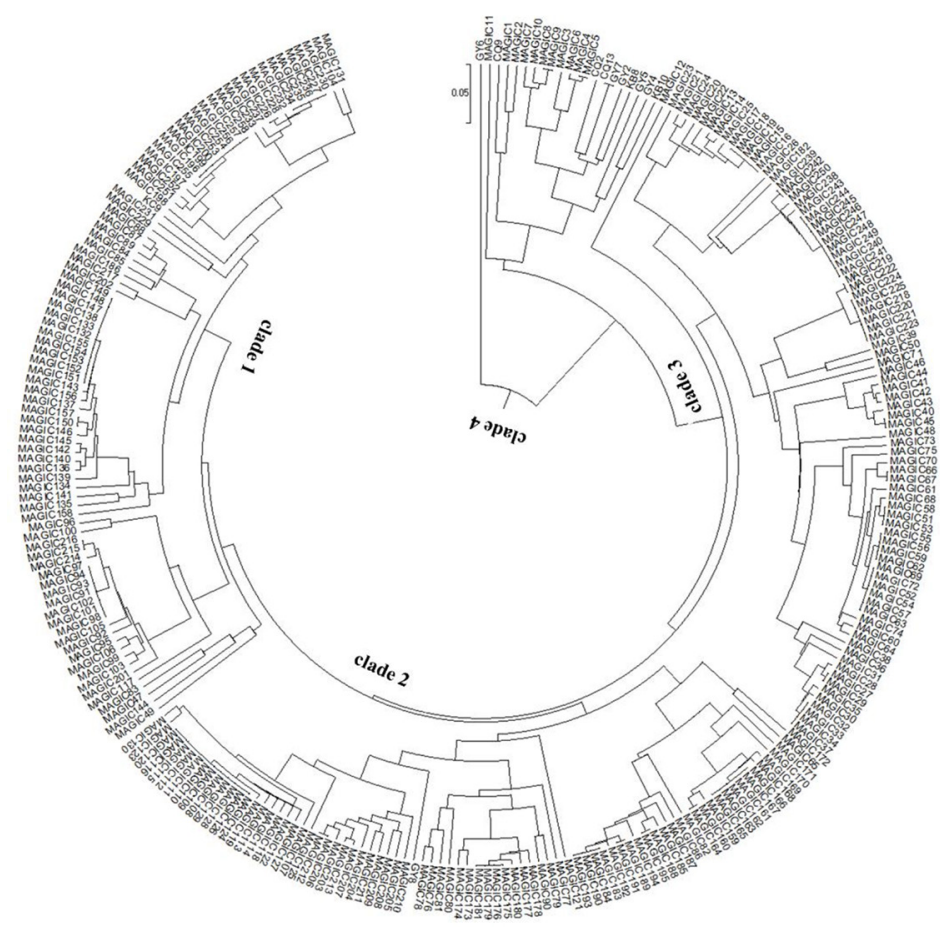

Figure 3. Genotype cluster analysis for the MAGIC population and the founders. The numbers represents different MAGIC population lines.

\section{DISCUSSION}

Upland cotton is mainly rooted in different upland cotton varieties, including Deltapine cotton, Stoneville, Delfos, Foster cotton, and King cotton, which are derived from twelve individual plants from one family in Mexico (Pan, 1998). This has led to a narrow genetic diversity of upland cotton varieties in China, and has resulted in barriers for breeding new cotton varieties (Pan, 1998). Therefore, it is necessary to improve the genetic diversity of the upland cotton population, e.g., by development of MAGIC populations. Previously, a new rice variety "Duo Ji Xin" has been developed using a MAGIC population (Li et al., 2013). "Duo Ji Xin" yields a comprehensive phenotype that has been approved by Hainan Province (Xu et al., 2014). The combined benefits of multi-genotype varieties for both yield and resistance improves their performance in favorable conditions, increases their stability under adverse conditions, and gives them an advantage over their component lines and even over monogenotype varieties under cultivation ( $\mathrm{Li}$ et al., 2013).

Each gene/QTL in a bi-parental population of RILs usually consists of two alleles and three genotypes. However, we detected more than two alleles in the MAGIC population derived from multiple parents. Kover et al. (2009) demonstrated that MAGIC populations enable the identification of novel QTLs with high precision in A. thaliana. Huang et al. (2011) used a public $A$. thaliana MAGIC population to test for complex traits, including QTL main effects and background interactions. Bi-parental RILs regularly display ordinary or bi-modal

Genetics and Molecular Research 15 (4): gmr15048759 
distributions in the frequency of quantitative or qualitative traits, whereas MAGIC populations display an even and continuous distribution that tends to deviate from their founders due to unequal contribution. Elucidation of both the founder lines and the resulting MAGIC population enables linkage and association analysis of genes/QTLs, especially in allotetraploid upland cotton. Until now, only one MAGIC population with four wheat founders has been reported for allopolyploid plants (Huang et al., 2012). The average PIC of upland cotton from different parental origins, breeding periods, and ecologies has been calculated using polymorphism analysis (Chen and Du, 2006). Similarly, the average PIC value of 32 main cotton cultivars was obtained using 26 polymorphic SSR markers (Zhang et al., 2012). In our study, genotype frequency, as a key index, indicated significant changes between the MAGIC population and the founder lines, indicating that the MAGIC population possesses abundant genetic diversity (Huang et al., 2015).

\section{Conflicts of interest}

The authors declare no conflict of interest.

\section{ACKNOWLEDGMENTS}

Research supported by the Special Fund for Agroscientific Research in the Public Interest (Grant \#201303008).

\section{REFERENCES}

Agarwal G, Sabbavarapu MM, Singh VK, Thudi M, et al. (2015). Identification of a non-redundant set of 202 in silico SSR markers and applicability of a select set in chickpea (Cicer arietinum L.). Euphytica 205: 381-394. http://dx.doi. org/10.1007/s10681-015-1394-3

Cavanagh C, Morell M, Mackay I and Powell W (2008). From mutations to MAGIC: resources for gene discovery, validation and delivery in crop plants. Curr. Opin. Plant Biol. 11:215-221.http://dx.doi.org/10.1016/j.pbi.2008.01.002

Chen G and Du XM (2006). Genetic diversity of source germplasm of Upland cotton in China as determined by SSR marker analysis. Yi Chuan Xue Bao 33: 733-745.http://dx.doi.org/10.1016/S0379-4172(06)60106-6

Churchill GA, Airey DC, Allayee H, Angel JM, et al.; Complex Trait Consortium (2004). The Collaborative Cross, a community resource for the genetic analysis of complex traits. Nat. Genet. 36: 1133-1137.http://dx.doi.org/10.1038/ ng1104-1133

Huang BE, George AW, Forrest KL, Kilian A, et al. (2012). A multiparent advanced generation inter-cross population for genetic analysis in wheat. Plant Biotechnol. J. 10: 826-839. http://dx.doi.org/10.1111/j.1467-7652.2012.00702.x

Huang BE, Verbyla KL, Verbyla AP, Raghavan C, et al. (2015). MAGIC populations in crops: current status and future prospects. Theor. Appl. Genet. 128: 999-1017. http://dx.doi.org/10.1007/s00122-015-2506-0

Huang X, Paulo MJ, Boer M, Effgen S, et al. (2011). Analysis of natural allelic variation in Arabidopsis using a multiparent recombinant inbred line population. Proc. Natl. Acad. Sci. USA 108: 4488-4493. http://dx.doi.org/10.1073/ pnas. 1100465108

Iqbal MJ, Aziz N, Saeed NA, Zafar Y, et al. (1997). Genetic diversity evaluation of some elite cotton varieties by RAPD analysis. Theor. Appl. Genet. 94: 139-144. http://dx.doi.org/10.1007/s001220050392

Kover PX, Valdar W, Trakalo J, Scarcelli N, et al. (2009). A Multiparent Advanced Generation Inter-Cross to fine-map quantitative traits in Arabidopsis thaliana. PLoS Genet. 5: e1000551.http://dx.doi.org/10.1371/journal.pgen.1000551

Li XF, Liu ZX, Lu DB, Liu YZ, et al. (2013). Development and evaluation of multi-genotype varieties of rice derived from MAGIC lines. Euphytica 192: 77-86. http://dx.doi.org/10.1007/s10681-013-0879-1

Li XF (2014). Method for constitution and production of multi-genotype colony varieties in crop plants. U.S. Patent No. $8,785,719$.

Pan JJ (1998). Cotton breeding. China Agricultural Press, Beijing.

Sallam A and Martsch R (2015). Association mapping for frost tolerance using multi-parent advanced generation inter-cross (MAGIC) population in faba bean (Vicia faba L.). Genetica 143: 501-514.http://dx.doi.org/10.1007/s10709-015-9848-z

Genetics and Molecular Research 15 (4): gmr15048759 
Smith JSC, Chin ECL, Shu H, Smith OS, et al. (1997). An evaluation of the utility of SSR loci as molecular markers in maize (Zea mays L.): comparisons with data from RFLPs and pedigree. Theor. Appl. Genet. 95: 163-173. http:// dx.doi.org/10.1007/s001220050544

Song GL, Cui RX, Wang KB, Guo LP, et al. (1998). A rapid improved CTAB method for extraction of cotton genomic DNA. Acta Gossypii Sin 10: 273-275.

Tu JL, Zhang MJ, Wang XQ, Zhang XL, et al. (2014). Genetic dissection of upland cotton (Gossypium hirsutum) cultivars developed in Hubei Province by mapped SSRs. Genet. Mol. Res. 13: 782-790. http://dx.doi.org/10.4238/2014. January. 31.4

Valdar W, Flint J and Mott R (2006). Simulating the collaborative cross: power of quantitative trait loci detection and mapping resolution in large sets of recombinant inbred strains of mice. Genetics 172: 1783-1797. http://dx.doi. org/10.1534/genetics.104.039313

Wang C, Fan JJ, Long LX, Yang JK, et al. (2016). Ecological response of rice multi-genotype variety in Dehong, Yunnan Province. Yunnan Nong Ye Da Xue Xue Bao 1: 1-6.

Wei $\mathrm{J}$ and $\mathrm{Xu} \mathrm{S}$ (2016). A random-model approach to QTL mapping in multiparent advanced generation intercross (MAGIC) populations. Genetics 202: 471-486.http://dx.doi.org/10.1534/genetics.115.179945

Xu CF, Li HJ and Li XF (2014). Breeding of multi-genotype variety Duojixin 3 and the description of its traits. China Rice 20: 71-73.

Xu SB, Tao YF, Yang ZQ and Chu JY (2002). [A simple and rapid methods used for silver staining and gel preservation]. Yi Chuan 24: 335-336.

Zhang YC, Yang WH, Kuang M, Xu HX, et al. (2012). Construction of DNA fingerprinting and analysis of genetic diversity with SSR markers for 32 leading cotton cultivars. Cotton Sci. 24: 120-126.

Genetics and Molecular Research 15 (4): gmr15048759 